\section{Implantación percutánea transeptal de válvula protésica aórtica sobre bioprótesis mitral degenerada}

\author{
GERMÁN ARMIJO ${ }^{1}$, ÁNGEL PUENTES ${ }^{1}$, ALEJANDRO CEBALLOS ${ }^{1}$, \\ MAURICIO QUEZADA ${ }^{1}$, CARLOS TAMAYO ${ }^{1}$, \\ HERNÁN DONOSO ${ }^{1}$, PABLO RAMÍREZ ${ }^{1}$, \\ CHRISTIAN BACKHOUSE ${ }^{1}$, LUIS NOMBELA-FRANCO ${ }^{2}$
}

\section{Percutaneous implantation of an aortic prosthetic valve on a degenerated mitral bioprosthesis. Report of one case}

Reintervention of a mitral degenerated bioprosthesis has a high surgical risk, especially in elderly patients with multiple comorbidities. We report a 74 years old female with two previous cardiac surgical procedures and a new structural mitral bioprosthesis deterioration with severe mitral regurgitation. Considering her high-surgical risk, a fully percutaneous treatment was performed with a balloon-expandable aortic valve in mitral position (valve-in-valve) through a transseptal approach with a favorable outcome. This technique is an attractive and effective option with a relatively low rate of complications that could solve this challenging and complex disease.

(Rev Med Chile 2021; 149: 137-141)

Key words: Heart Valve Prosthesis; Mitral Valve Insufficiency; Transcatheter Aortic Valve Replacement.
${ }^{1}$ Centro de Enfermedades

Cardiovasculares. Hospital San Juan de Dios. Santiago, Chile. ${ }^{2}$ Instituto Cardiovascular. Hospital Clínico San Carlos. IdISSC. Madrid, España.

Conflictos de interés: El Dr. Luis Nombela-Franco ha recibido honorarios de Edwards Lifesciences y Abbott por conferencias.

Trabajo no recibió financiamiento.

Recibido el 13 de agosto de 2020, aceptado el 16 de noviembre de 2020 .

Correspondencia a: Dr. Germán Armijo Ortiz Centro de Enfermedades Cardiovasculares. Hospital San Juan de Dios. Huérfanos 3255. CP 8350488 Santiago, Chile. garmijor@hotmail.com
$\mathrm{E}$ 1 deterioro estructural de una bioprótesis quirúrgica en posición mitral es un fenómeno frecuente, estimándose que cerca de $40 \%$ de los pacientes requerirán una reintervención dentro de los siguientes 15 años tras su cirugía ${ }^{1}$, con un elevado riesgo asociado de morbilidad y mortalidad $^{2}$. La necesidad de disponer de tratamientos cada vez menos invasivos para abordar la válvula mitral ha permitido el desarrollo de diferentes técnicas durante los últimos años, destacando el implante valvular transcatéter sobre bioprótesis mitral degenerada ("Valve-in-valve" $(\mathrm{ViV}))$. A continuación describimos, según nuestro conocimiento, el primer implante valvular completamente percutáneo sobre una bioprótesis mitral por abordaje transeptal realizado en nuestro país.

\section{Caso clínico}

Mujer de 74 años con antecedentes de fibrilación auricular permanente y cardiopatía reumática sometida a cirugía de reemplazo mitral mecánico el año 2000 con prótesis monodisco Medtronic Hall (Medtronic Inc., Minnesota, EE. UU.) y posteriormente recambio mitral con bioprótesis Carpentier Edwards No31 (Edwards Lifesciences, Inc., Irvine, California, EE. UU.) en el año 2009 debido a trombosis aguda protésica en relación a tiempos de coagulación sub-terapéuticos.

Ingresa a nuestro centro asistencial tras un cuadro de semanas de evolución caracterizado por deterioro de su clase funcional, tos, sensación febril y disnea a pequeños esfuerzos, demostrando en sus pruebas complementarias elevación ligera 
de sus parámetros inflamatorios, condensación del lóbulo superior derecho en la radiografía de tórax con hemocultivos seriados negativos. Los ecocardiogramas transtorácico y transesofágico mostraron una función ventricular izquierda conservada, hipertensión pulmonar moderada e insuficiencia mitral (IM) severa excéntrica, central, secundaria a prolapso de uno de los velos, descartando la presencia de trombos o infecciones concomitantes, recibiendo tratamiento inicialmente con antimicrobianos, ventilación mecánica no invasiva y terapia depletiva con evolución regular. Su caso fue discutido en el equipo multidisciplinario y considerando el alto riesgo quirúrgico de una tercera cirugía cardíaca (riesgos de mortalidad estimados en $10,0 \%$ y $12,6 \%$ mediante Euroscore-2 y STS Score, respectivamente) se planteó como primera opción el tratamiento completamente percutáneo por vía transeptal con implantación de una válvula balón expandible Sapien-3 29 mm (Edwards Lifesciences, Inc., Irvine, California, EE. UU.). La valoración pre operatoria incluye una tomografía computarizada (TC) car- díaca sincronizada para determinar el diámetro interno real de la prótesis $(26,6 \mathrm{~mm})$ junto con la angulación mitro-aórtica (117\%), estimación del área del nuevo TSVI $\left(199 \mathrm{~mm}^{2}\right)$, y la simulación de implante de la prótesis percutánea (Figura 1). Se obtuvo consentimiento informado para la presentación de este caso.

\section{Descripción del procedimiento}

El procedimiento se realizó con anestesia general y guiado por ecografía transesofágica. Tras utilizar un pre-cierre vascular con dispositivo Proglide (Abbott Vascular Inc., Santa Clara, CA, USA), se accedió a la aurícula izquierda tras punción transeptal permitiendo el avance de vaina deflectable Mobicath 8,5Fr orientada hacia el anillo mitral. A través de este sistema se avanzó un catéter Pigtail 5Fr hacia el ápex del ventrículo izquierdo para la inserción de una guía de alto soporte $\mathrm{Safari}^{2}$ XS (Boston Scientific, Boston, MA, USA).

a dicho nivel, para realizar posteriormente una
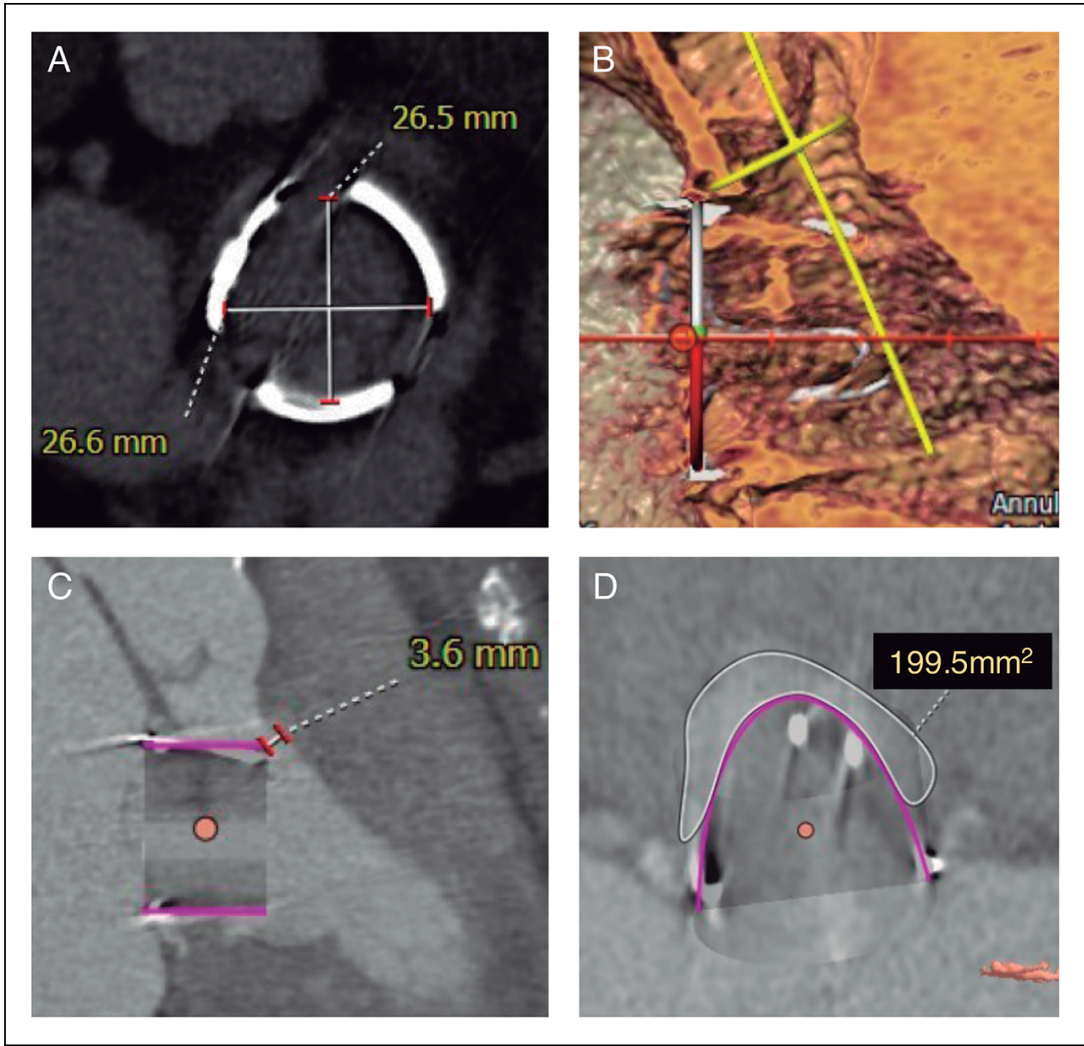

Figura 1. Análisis de la tomografía computarizada previo al implante. (A) diámetros internos de la prótesis, (B) angulación mitro-aórtica, (C y D) estimación del diámetro y área del neo-LVOT post implante $\left(3,6 \mathrm{~mm}\right.$ y $199,5 \mathrm{~mm}^{2}$, respectivamente). LVOT $=$ Tracto de salida del ventrículo izquierdo. 


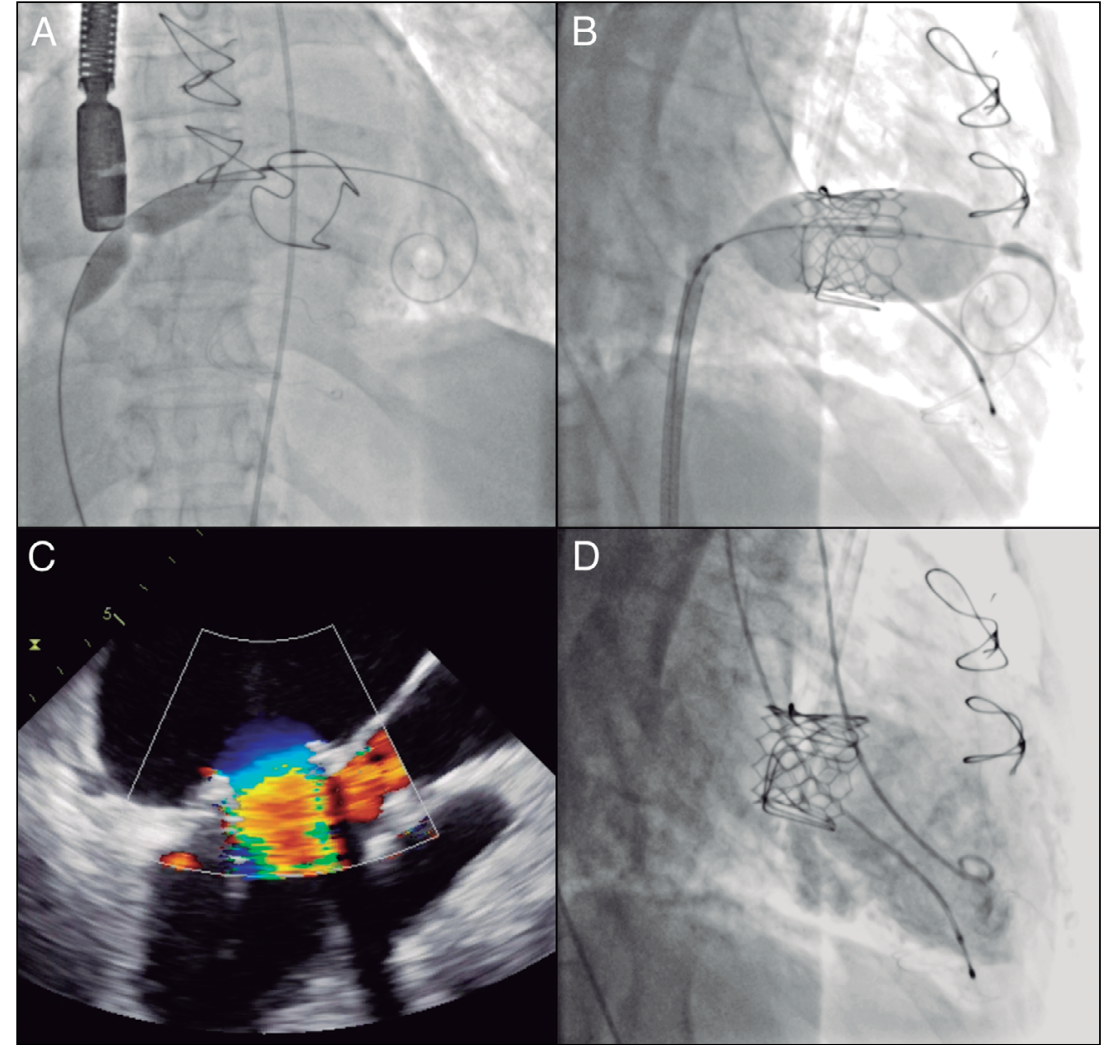

Figura 2. Implante percutáneo transeptal de prótesis aortica balón expandible en posición mitral. (A) Septostomía con balón para facilitar el cruce, (B) implante de prótesis balón expandible, (C y D) imagen ecográfica (eje $130^{\circ}$ ) y angiográfica final con ausencia de insuficiencia mitral residual. septostomía con balón Atlas-PTA Medical de 12 $\mathrm{mm}$ (Figura 2A). A través del introductor eSheath $16 \mathrm{~F}$ (Edwards Lifesciences, Inc, Irvine, California, EE.UU), se avanzó el sistema de liberación con la orientación del sistema de flexión para el acceso mitral (logo de Edwards hacía las $6 \mathrm{~h}$ ), junto con la prótesis Edwards Sapien-3 29 mm montada en el sentido opuesto de la posición aórtica.

Una vez en la vena cava inferior se acopla el balón sobre la prótesis, para avanzar todo el sistema con facilidad hacia la aurícula izquierda hasta posicionar la válvula a nivel de la bioprótesis degenerada. Una vez retirado el "empujador", y bajo estimulación ventricular rápida a $160 \mathrm{lpm}$ mediante sonda de marcapasos por vía yugular derecha, se procede al inflado (volumen nominal) e implante exitoso de la válvula con expansión adecuada (Figura 2B). La ecografía transesofágica y la angiografía confirmaron ausencia de IM residual, gradientes intraprotésicos y del TSVI normales (Figuras 2C y 2D, respectivamente). La evolución posterior fue satisfactoria, siendo dada de alta al cuarto día post procedimiento con anticoagulación oral mediante antagonista de vitamina K. A 3 meses de seguimiento la paciente se mantenía en clase funcional NYHA I sin eventos clínicos mayores.

\section{Discusión}

La necesidad de re-intervenir quirúrgicamente la válvula mitral es un procedimiento que conlleva un alto riesgo peri-operatorio, con una mortalidad precoz cercana al $\sim 20 \%$ a partir de una tercera cirugía ${ }^{3}$. Por ello, el implante valvular mitral transcatéter ha surgido como una alternativa a la cirugía en pacientes de alto riesgo con disfunción severa de una bioprótesis degenerada. Este procedimiento, comúnmente llamado "Valve-in-Valve mitral", se ha realizado con prótesis aórtica transcatéter de la familia Edwards SAPIEN, recibiendo la indicación en Junio del 2017 por la Food and Drug Administration (FDA) para tratar prótesis 
biológicas mitrales disfuncionantes. Además su uso se ha expandido a pacientes con anuloplastia mitral ("Valve-in-Ring") o anillo mitral severamente calcificado ("Valve-in-MAC") 4,5. Inicialmente el abordaje transapical fue mayoritario por experiencia previa con la TAVI por esta vía y su óptima coaxialidad con el plano mitral. Sin embargo, la necesidad de toracotomía, trauma del ápex, y los peores resultados de esta vía en la TAVI, ha generado un número creciente de implantes por vía transeptal con resultados comparables o incluso superiores al abordaje transapical ${ }^{4,6,7}$.

Una correcta planificación es crucial para asegurar el éxito del procedimiento y evitar complicaciones. Se debe prestar especial atención en estos procedimientos a la posible obstrucción del TSVI con el TC cardíaco previo, que permite evaluar el diámetro interno real de las prótesis degenerada, tamaño de la cavidad ventricular izquierda y TSVI, ángulo mitro-aórtico (mayor riesgo frente a ángulos más agudos) y finalmente la estimación virtual del área TSVI posterior a la simulación del implante de la prótesis requerida ("neo-LVOT"). El valor de corte para el neo-LVOT no está completamente definido, pero publicaciones recientes señalan un mayor riesgo de obstrucción con áreas inferiores a $170 \mathrm{~mm}^{2}{ }^{[8]}$, pudiendo plantearse en este grupo de paciente de riesgo alternativas como la alcoholización septal ${ }^{9} \mathrm{o}$ la laceración percutánea del velo anterior de válvula mitral ${ }^{10}$.

Recientemente han sido publicados los resultados a 1 año del mayor registro prospectivo de pacientes de alto riesgo quirúrgico con bioprótesis mitral degenerada $(\mathrm{n}=1.529)$ sometidos a ViV mitral con la prótesis Edwards Sapien-3, comparando resultados según abordaje transeptal (TS, $86,7 \%$ del total) vs transapical (TA $)^{7}$. La tasa de éxito global fue de $96,8 \%$, con una baja incidencia de complicaciones (accidente cerebrovascular 0,7\%, embolización del dispositivo $0,3 \%$, obstrucción del TSVI $0,9 \%$ y perforación cardíaca en $1.1 \%$, similar para ambos grupos). El grupo TA mostró una mayor mortalidad cardiovascular intrahospitalaria y días de estancia comparado al grupo TS $(4,4 \%$ vs $1,8 \%$; $p=0,03$ y 6 [3-9] vs 2 [1-5] días; $\mathrm{p}<0,001$, respectivamente). Los resultados a 30 días revelaron una mortalidad global de $5,4 \%$, inferior a $11,1 \%$ de lo reportado sobre 1096 pacientes reintervenidos quirúrgicamente de una prótesis mitral en el registro de la Sociedad de Cirujanos Torácicos (STS) de Estados Unidos de
Norteamérica ${ }^{11}$. Finalmente, el grupo TS se asoció a una menor mortalidad global a 1 año $(15,8 \%$ vs $21,7 \% ; p=0,03)$, con un perfil hemodinámico de la válvula satisfactorio para ambas ramas (gradiente medio de 7,0 $\pm 2,89 \mathrm{mmHg}$ a 1 año).

\section{Conclusión}

El implante valvular mitral transcatéter por vía transeptal en pacientes con prótesis mitral previa surge como una técnica alternativa y menos invasiva que el recambio mitral quirúrgico, pudiendo ser realizado de forma segura y con una tasa de complicaciones baja, gracias a una planificación preoperatoria adecuada.

\section{Referencias}

1. Bourguignon $T$, Bouquiaux-Stablo A-L, Loardi C, Mirza A, Candolfi P, Marchand M, et al. Very late outcomes for mitral valve replacement with the Carpentier-Edwards pericardial bioprosthesis: 25-year follow-up of 450 implantations. J Thorac Cardiovasc Surg 2014; 148: 2004-11.el. https://doi.org/10.1016/j. jtcvs.2014.02.050.

2. Vohra HA, Whistance RN, Roubelakis A, Burton A, Barlow CW, Tsang GMK, et al. Outcome after redo-mitral valve replacement in adult patients: a 10 -year single-centre experience. Interact Cardiovasc Thorac Surg 2012; 14: 575-9. https://doi.org/10.1093/icvts/ivs005.

3. Expósito V, García-Camarero T, Bernal JM, Arnáiz E, Sarralde A, García I, et al. Repeat mitral valve replacement: 30-years' experience. Rev Esp Cardiol 2009; 62: 929-32. https://doi.org/10.1016/s1885-5857(09)72658-1.

4. Guerrero M, Vemulapalli S, Xiang Q, Wang DD, Eleid M, Cabalka AK, et al. Thirty-Day Outcomes of Transcatheter Mitral Valve Replacement for Degenerated Mitral Bioprostheses (Valve-in-Valve), Failed Surgical Rings (Valve-in-Ring), and Native Valve With Severe Mitral Annular Calcification (Valve-in-Mitral Annular Calcification) in the United States. Circ Cardiovasc Interv 2020. https://doi.org/10.1161/CIRCINTERVENTIONS.119.008425.

5. Guerrero M, Salinger M, Pursnani A, Pearson P, Lampert M, Levisay J, et al. Transseptal transcatheter mitral valve-in-valve: A step by step guide from preprocedural planning to postprocedural care. Catheter Cardiovasc Interv Off J Soc Card Angiogr Interv 2018; 92: E185-96. https://doi.org/10.1002/ccd.27128. 
6. Dvir D, Webb J. Mitral valve-in-valve and valve-in-ring: technical aspects and procedural outcomes. EuroIntervention J Eur Collab Work Group Interv Cardiol Eur Soc Cardiol 2016; 12: Y93-6. https://doi.org/10.4244/ EIJV12SYA25.

7. Whisenant B, Kapadia SR, Eleid MF, Kodali SK, McCabe JM, Krishnaswamy A, et al. One-Year Outcomes of Mitral Valve-in-Valve Using the SAPIEN 3 Transcatheter Heart Valve. JAMA Cardiol 2020. https://doi. org/10.1001/jamacardio.2020.2974.

8. Yoon S-H, Bleiziffer S, Latib A, Eschenbach L, Ancona $\mathrm{M}$, Vincent F, et al. Predictors of Left Ventricular Outflow Tract Obstruction After Transcatheter Mitral Valve Replacement. JACC Cardiovasc Interv 2019; 12: 182-93. https://doi.org/10.1016/j.jcin.2018.12.001.

9. Deharo P, Urena M, Himbert D, Brochet E, Rouleau
F, Pinaud F, et al. Bail-Out Alcohol Septal Ablation for Left Ventricular Outflow Tract Obstruction After Transcatheter Mitral Valve Replacement. JACC Cardiovasc Interv 2016; 9: e73-6. https://doi.org/10.1016/j. jcin.2016.01.010.

10. Khan JM, Babaliaros VC, Greenbaum AB, Foerst JR, Yazdani S, McCabe JM, et al. Anterior Leaflet Laceration to Prevent Ventricular Outflow Tract Obstruction During Transcatheter Mitral Valve Replacement. J Am Coll Cardiol 2019; 73: 2521-34. https://doi.org/10.1016/j. jacc.2019.02.076.

11. Mehaffey HJ, Hawkins RB, Schubert S, Fonner C, Yarboro LT, Quader M, et al. Contemporary outcomes in reoperative mitral valve surgery. Heart $\mathrm{Br}$ Card Soc 2018; 104: 652-6. https://doi.org/10.1136/heartjnl-2017-312047. 